\title{
Elephant Intrusion Detection and Repulsion System using Matlab
}

\author{
DR.M.Senthamil selvi, S. Jansi Rani, Karthiga.T, Kamala.PL, Kanishka.V R
}

\begin{abstract}
Elephant intrusion across the railway track leads to HumanElephant conflict, collisionof trains, elephant death and injuries. Railway tracks pass through wild life habitats in several Indian states. In India, due to railway cross lines, the accidents that resulted in the death of 249 elephants during 1987-2018.The surveillance and monitoring the elephants in the railway line in the forest is very difficult. The speed of the train in India among forest areas is around $50-55 \mathrm{~km} / \mathrm{h}$ during day and $35 \mathrm{~km} / \mathrm{h}$ at night, the engine driver can't stop the train once the elephant is seen. So, we develop a system to detect the elephant intrusion at a certain distance by using sensors and image processing using MATLAB, creates an alert to engine driver and forest officer of the region and repel the elephant from the railway line. Hence we are creating a prototype model for real time interaction of elephant intrusion across railway tracks.
\end{abstract}

Key Terms: Railway tracks, Sensors, Image Processing,MATLAB

\section{INTRODUCTION}

Elephant Intrusion system detect the elephant on the track and alerts the engine driver that the elephant is on the track, which reduces the elephant conflict and make the elephant to move away from the track. The main aim of the system is to reduce the elephant conflict across the railway track. In this system we use transmitter and receiver. The transmitter and receiver starts to communicate once the train starts to move, this communication take place only at the moment of the train. Arduino act as a microcontroller which control all part of the system. Here, we are developing prototype model for real-time elephant intrusion and a design to spot the elephant intrusion. Here we are using IOT and image processing concepts to detect the elephant on the track, the ultrasonic sensor is used to detect the object in front of the sensor, along with that LCD is used to determine the distance of the object. Buzzer alarm make a huge alarm to make people around the village to understand elephant is on the track, and the alarm sound also make elephant to move away from the track. After the object detection by the sensor, system automatically on the webcam and identify whether object is elephant, if the object is elephant, it automatically send a sms through gsm to the engine driver that a elephant is detected. Here we are using an image processing technique to detect whether the object detected is elephant or not.Matlab concepts is used to identify the elephant object in front of the train.

Revised Manuscript Received on May 27, 2020.

* Correspondence Author

DR.M.Senthamil selvi*, Professor \& Head in the Department of Information Technology at Sri Ramakrishna engineering college, Coimbatore.

Mrs. S. Jansi Rani, Assistant Professor, Department of Information Technology, Sri Ramakrishna Engineering College,Coimbatore.

Karthiga.T , B.TECH Information Technology, Sri Ramakrishna Engineering College, Coimbatore.

Kamala.PL , B.TECH Information Technology, Sri Ramakrishna Engineering College, Coimbatore.

Kanishka.V R , B.TECH Information Technology, Sri Ramakrishna Engineering College, Coimbatore.

(C) The Authors. Published by Blue Eyes Intelligence Engineering and Sciences Publication (BEIESP). This is an open access article under the CC BY-NC-ND license (http://creativecommons.org/licenses/by-nc-nd/4.0/)

\section{Description Of Proposed System Components}

\section{ArduinoUNO}

ArduinoUNO is a microcontroller based on ATmega328, ArduinoUNO is powered via USB connection, It has digital inputs, analog inputs, crystal oscillator. ArduinoUNO is open source platform, based on easy to use hardware and software. It differ from all boards in that they do not use FTDIUSB-to-serial converter. Instead, it is programmed as a USB-to-Serial connector.

The Arduino UNO can be programme with Arduino software.

The Arduino has number of way to communicate with computer, other micro controller. They operate at 5 volts. Lead from the battery connected in Vin or Gnd of pin header of power connecter. Arduino UNO has a serial monitor.

\section{Encoder with RFTransmitter}

In RF Transmitter receives serial data and transmit it wirelessly through its RF through its antenna, RF transmitter is the small pcb assembly capable of transmitting the radio wave to carry data. Transmitter generates a radio frequency current applied to antenna, which in turn radiate radio waves. Transmitter generates radio waves for communication, radar and navigational purpose.

LC filter section is given to modulated signal. After the filtration is done, antenna is used to transmit the RF modulated signal. This is a low power and high noise immunity CMOS technology. Data code as positive polarity, RFTransmitter are usually subject to regulatory requirements which dictate the band edge

\section{Decode with RFReceiver}

In RF Receiver receives the modulated RF signal, and demodulates it, similar to transmitter, hear also we have two push buttons and one for selection of code and receiving the data. Once the data is received by the receiver module that data is demodulated and goes to the receiver pin. Receiver will select the signal of interest and reject all others and it will amplify the received signal to a useful level. It will demodulate the intelligence that was carried by the radio signal. Some kind of FM Receiver a similar feedback loop can be used to fined tune the LO to ensure that received signal is dead centre in the channel

\section{Ultrasonic Sensors}

Ultrasonic Sensor is used to identify the distance of the object. It offer high accuracy and stable reading. It as both receiver and transmitter. When the transmitter send signal, when the signal find the object it receive it back, the time between the transmission and reception give the distance of the object. Microcontroller is used for communication with an ultrasonic sensor. Sensor generates eight acoustic wave bursts and initiates a time counter. The output of the ultrasonic sensor is high pulse with the same duration as the time difference between transmitted ultrasonic bursts and received echo signal.
Blue Eyes Intelligence Engineering \& Sciences Publication (C) Copyright: All rights reserved.

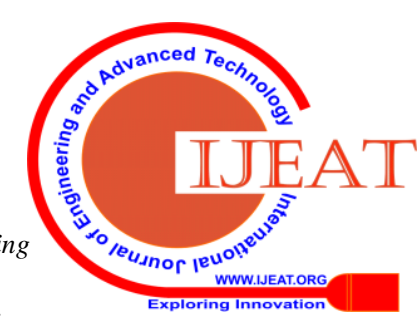




\section{GSM}

GSM modem is a interface. This is used to send sms and operates over a subscription to a mobile operator, just like a mobile phone.

It is a wireless modem that sends and receives data through radio waves.gsm has wide range of applications in transaction terminals, weather stations etc. Modem low power consumption of 0.25.can be used for gsm based voice communication and network LEDs for easy debugging.

\section{Alarm}

Buzzer is a small yet efficient component to add sound features to our system. It can provide an effective way of interacting with users or raising an alarm, portable equipments due to its compact size. This is an electromagnetic system. This was identical to metal bell without a metal gong. Small sound module determine by Arduino output pin, with the Arduino tone library, many different sounds can be made.

\section{LCD}

LCD available to display arbitrary images, this is a panel display or visual display uses the properties of liquid crystal. This is a fixed image with low information content and 7 segment displays as in digital clock images are made up of Large number of small pixels, they are used in many applications and have replaced cathode ray

tube displays in nearly all applications. They are susceptible to image persistence

\section{WebCamera}

Web camera are small device sit on a desk, that stream video on real time. Resolution of webcam is low. They receive and control data via internet. They are relatively inexpensive compare to other cams. They can easily identify front object, they connected to computer via USB.They use make remote work possible. Webcam have at least 30 frames per seconds. Webcam have a built in microphone and the mic depend on the type of video.

\section{RELATEDWORK}

Elephant intrusion system is mainly concern towards the countries have more number of elephants population. So many strategies are followed to control the human elephant conflict. We also need knowledge to find the solution for the human elephant conflict

L.JenShaffer [1] developed and followed certain strategies to prevent human-elephant conflict. Exclusionary methods followed for human-elephant conflict prevention were by developing electric fences and trenches around agricultural fields and other methods like acoustic deterrents by using fire crackers and throwing stones on them or throwing some metal objects.

Radhika.M[2] developed a digital solution by introducing the technology Internet of Things By using these technology we can alert the local people about the elephant moment around the area of the forest by detecting the infrasound communication of elephants and warning is given through android based application

Mohamed Fazil Mohamed Firdhous [3] Presented design and prototype implementation of smart elephant detection system, this system will alerts the human settlements when the elephant approaches. The architecture of the system involves microcontroller,localstorage ,communicatio $\mathrm{n}$ module, sensor panels and the power controller. Sensor is used in detecting the ground waves.

J.P.Dominguez-Morales [4] proposed a

system based on wireless sensor network installed in any of the national park, to gather the information related to all animals' they are based on they are neural network implementation to classify their behavior based on sensitive information gathered. Once a behavior is detected, the network redirects this information to an external part of the database for further Implementation. This is the solution reduces power consumption and facilitates animals' behavior monitoring for biologists

\section{PROPOSED METHODOLOGY}

Elephant Intrusion Detection and Repulsion System in the railway tracks is developed to avoid Human-Elephant intrusion and life damages. This is the system to detect elephant in real time and try to repel the elephant away from railway track without human interference.

\section{Microcontroller and Sensor}

The hardware components used are microcontroller [ArduinoUNO] and sensor [Ultrasonic sensor]. When the RF receiver receives signal from the RFtransmitter, which indicates that the train gets started which runs through the particular track. The sensor detects the moving object around the range and the distance between the object and the track is displayed in the LCD screen.

\section{MATLAB}

MATLAB is the software which is used. It is a high performance language for technical computing. When the sensor detects an object the webcam takes the image of the object and using image processing technique the object is tested for the elephant. In image processing, median filter is used to reduce noise and preserve edges. HSV segmentation is used as an color detection algorithm searches an image for pixels. Multiclass Support vector machine is used to analyze data for classification and regression analysis.

\section{Workflow of proposed system}

The workflow of the proposed Elephant intrusion detection and repulsion system involves the following steps:

Step 1:The RF receiver receives the signal From the RF transmitter indicates train gets started.

Step 2: The sensor detects the object which is moving in the particular area and the distance is shown in the LCD screen.

Step 3: The webcam captures the image of the object.

Step 4: The captured image is tested for

Elephant

Step 5: If the object is an elephant then alert message is sent to engine driver and the forest officer.

Step 6: And the buzzer is on to repel the elephant away from the area.

\section{RESULTS AND OUTPUT}

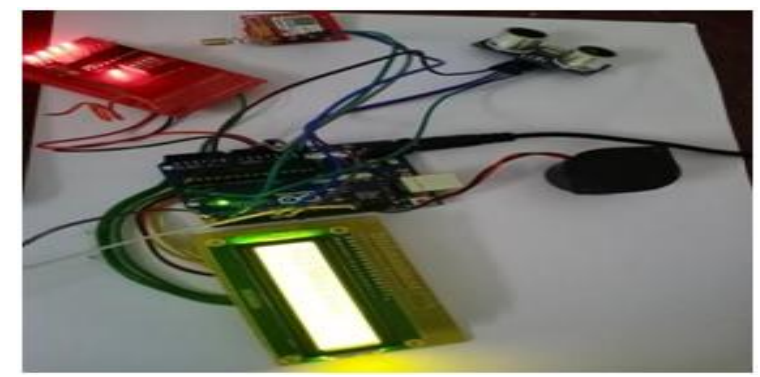

Fig-1: Working of hardware components.

Blue Eyes Intelligence Engineering

\& Sciences Publication

(C) Copyright: All rights reserved. 


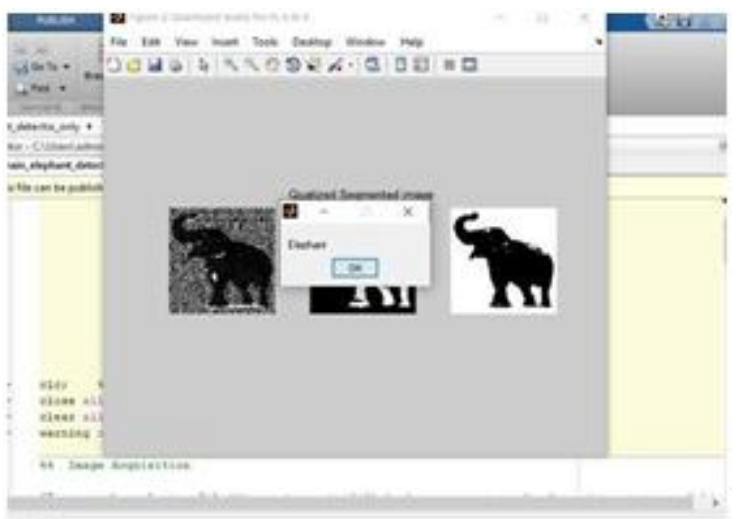

Fig-2: The object is detected as an Elephant.

\section{Mar 14, 14:36 D \\ ELEPHANT DETECTED IN RAILWAY TRACK}

Fig-3: The alert message sent by the GSM

This Elephant Intrusion Detection and Repulsion System is used to detect the intrusion of elephants, to alert and to repel. When the sensor detects an object, the webcam is used to capture the image of the object and by using image processing algorithm we can predict the elephant. Once the elephant is detected an GSM message is sent to the engine driver and the forest officer of the particular area and the elephant repelled into the forest by using buzzer.

\section{CONCLUSION}

Here we are developing a prototype model to prevent elephant intrusion in railway tracks and alert the engine driver to stop the train and the forest officer to rescue the elephant if the elephant is not repelled into the forest by the buzzer. Hence we are using Webcam; we can have a real time interaction with the environment.

\section{REFERENCES}

1. L.JenShaffer,JamonVanDenHoek,KapilK.Khadh a,andKusumJ.Naithani,"Human-ElephantConflict:A Review of curren Management Strategies and Future Directions'Frontiers in Ecology and Evolution,6,235,Jan 2019.

2. Radhika.M,RahamathunNashreen.Z and Vinjam LakshmiSravya,' Elephant warning system using IoT",International Research Journal of Engineering and Technology,2018.

3. MohamedFazil,MohamedFirdhous,'IoT Enabled Smart Elephant Detection System for Combating Human Elephant Conflict",IEEEConference, Dec2018. J.P.DominguezMorales,A.RiosNavarro,M.Domi Morales,R TapiadorMorales,D.GutierrezCaballero,A.Jimenez- $\quad$ Fernandez,A.LinaresBarranco,’WirelessSensor NetworkforWildlifeTrackingandBehaviourClassifi cationof AnimalsinDonana”,IEEE Communication Letters,22 Sep 2016.

4. R.Maheswari,'Developmentof Embedded Based System to Monitor Elephant Intrusion in ForestBorderAreasUsing Internet of Things",International Journal of Engineering Research,1 Jul 2016.

5. M.S.Nakandala,S.S.Namasivayam

6. .Chandima,'Detecting Wild Elephants ViaWSN for Early Warning System", International Research JournalofEngineeringandTechnology.

7. CharithaElvitigala,ErandaTennakoon,"Towards A Sensor System to Tame the Human Elephant Conflict",IEEEConference,2015.

8. N.Suganthi,N.Rajathi,FarithulInzamamM," ElephantIntrusion Detection and Repulsion TechnologyandEngineering,Nov2018.

9. R.Maheswari,'Development ofEmbedded Based System to Monitor
Elephant Intrusion in Forest Border Areas Using Internet of Things", International Research Journal of Engineering and Technology,2016.

10. S.J.Sugumar,R.Jayaparvathy,"AnImproved Real Time Image Detection System for Elephant Intrusion along the Forest Border Areas", The Scientific WorldJournal,2014.

\section{AUTHORS PROFILE}

DR.M.Senthamil selvi is currently working as Professor \& Head in the Department of Information Technology at Sri Ramakrishna engineering college, Coimbatore. She has 23 years of teaching experience and 3 years of industry experience.

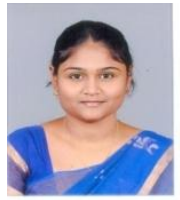

Mrs. S. Jansi Rani is currently working as assistant professor in the department of information technology at Sri ramakrishna engineering college,Coimbatore. She has 7 Years of teaching experience.

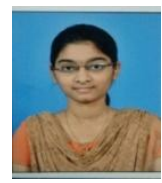

Karthiga.T is currently studying B.TECH information technology at Sri Ramakrishna engineering college, Coimbatore.

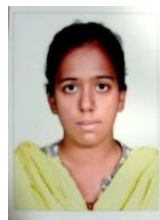

Kamala.PL is currently studying B.TECH information technology at Sri Ramakrishna engineering college, Coimbatore.

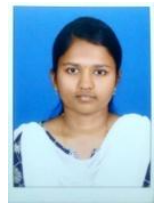

Kanishka.V R is currently studying B.TECH information technology at Sri Ramakrishna engineering college, Coimbatore.

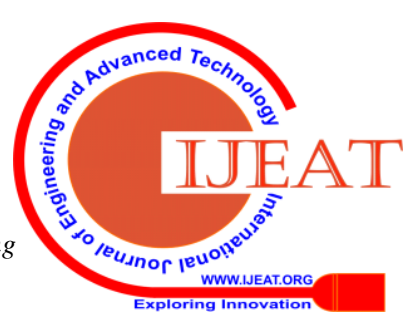

